L. SARIO

KŌDAI MATH. SEM. REP.

26 (1974), 53-57

\title{
QUASIHARMONIC DEGENERACY OF RIEMANNIAN $N$-MANIFOLDS
}

\author{
By Leo SARIo
}

The harmonic classification of Riemannian manifolds of higher dimension was recently brought to completion by the construction of manifolds which carry bounded but no Dirichlet finite nonconstant harmonic functions (Kwon [2], Hada-Sario-Wang [1]). In contrast, almost nothing is known about the quasiharmonic classification of higher dimensional manifolds. The purpose of the present study is to establish inclusion relations constituting such a classification.

Let $Q$ be the class of quasiharmonic functions [6], i. e., solutions of $\Delta u=1$, where $\Delta=d \delta+\delta d$ is the Laplace-Beltrami operator. Denote by $Q P, Q B, Q D$, and $Q C$ the classes of quasiharmonic functions which are positive, bounded, Dirichlet finite, and bounded Dirichlet finite, respectively. The notation $\widetilde{O}_{Q X}^{N}$, $\mathcal{O}_{Q X}^{N}$, with $X=P, B, D$, or $C$, will be used for the classes of Riemannian manifolds $M$ of dimension $N>2$ for which $Q X(M)=\emptyset$ or $\neq \emptyset$, respectively. The class of parabolic $N$-manifolds, characterized by the nonexistence of Green's functions, is designated by $O_{G}^{N}$. We shall prove that the complete classification scheme

$$
\mathcal{O}_{G}^{N}<\mathcal{O}_{Q P}^{N}<\mathcal{O}_{Q B}^{N} \cap \mathcal{O}_{Q D}^{N}<\mathcal{O}_{Q B}^{N}, \mathcal{O}_{Q D}^{N}<\mathcal{O}_{Q B}^{N} \cup \mathcal{O}_{Q D}^{N}=\mathcal{O}_{Q C}^{N}
$$

holds for every $N$. Here "<" signifies strict inclusion and “ $\Theta_{Q B}^{N}, \mathcal{O}_{Q D}^{N}$ ” means that the inequalities are valid for both classes.

1. We shall cover the relations in the order given above. Regarding $\mathcal{O}_{G}^{N}$ and $\mathcal{O}_{Q P}^{N}$, every $u \in Q P$ is superharmonic by $\Delta u=1$ and its existence therefore implies that of the Green's functions (see e.g. [7]). To prove $\mathcal{O}_{G}^{N}<\mathcal{O}_{Q P}^{N}$, it thus suffices to find a hyperbolic $N$-manifold with $Q P=\emptyset$. The trivial example of the Euclidean $N$-space does not qualify for $N=2$, whereas the following example is valid for all $N$. It is an $N$-torus cut open along a pair of opposite faces and equipped with a suitable metric.

LEMMA 1. Let $T$ be the space

$$
0<x<1, \quad\left|y_{i}\right| \leqq 1, \quad i=1, \cdots, N-1,
$$

Received April 2, 1973.

The work was sponsored by the U.S. Army Research Office-Durham, Grant DAARO-D-31-124-71-G181, University of California, Los Angeles. 
with each face $y_{i}=1$ identified with the opposite face $y_{i}=-1$ by parallel transla. tion. Endow $T$ with the metric

$$
d s^{2}=\left(x^{-2}+\left((1-x)^{-2}\right) d x^{2}+\left(x^{-2}+(1-x)^{-2}\right)^{1 /(N-1)} \sum_{i=1}^{N-1} d y_{i}^{2} .\right.
$$

Then $T \in \tilde{\mathcal{O}}_{G}^{N} \cap \mathcal{O}_{Q P}^{N}$.

Proof. The metric tensor has the determinant $g=\left(x^{-2}+(1-x)^{-2}\right)^{2}$, and the first entry of the conjugate tensor is $g^{x x}=\left(x^{-2}+(1-x)^{-2}\right)^{-1}$. Characterization of an $h(x)$ in the class $H$ of harmonic functions,

$$
\Delta h=-g^{-\frac{1}{2}}\left(g^{\frac{1}{2}} g^{x x} h^{\prime}\right)^{\prime}=0,
$$

gives $h^{\prime \prime}=0$, that is, $h=a x+b$. Therefore, the harmonic measure of the ideal boundary of $T$ is positive, which entails $T \in \widetilde{\mathscr{O}}_{\tilde{G}}^{N}$ (see e. g. [7]).

We recall that a manifold $M$ belongs to $\tilde{\mathscr{O}}_{Q P}, \widetilde{\mathcal{O}}_{Q B}$, or $\widetilde{\mathscr{O}}_{Q D}$ according as the potential

$$
G 1(p)=\int_{M} g(p, q) d q
$$

exists or is bounded at some, and hence at every, point $p \in M$, or has a finite Dirichlet integral

$$
D(G 1)=G(1,1)=\iint_{M \times M} g(p, q) d p d q,
$$

respectively (Nakai-Sario [3]). Here $g$ is the Green's function with pole $q$, and $d p, d q$ are the volume elements at $p$ and $q$.

For the function

$$
u(x)=\log x+\log (1-x)
$$

we obtain by direct computation $\Delta u=1$. If $G 1$ exists, then by the symmetry of $T$ with respect to all $y_{i}$-coordinates, $G 1$ is a function of $x$ only. Since $\Delta G 1=1$, we have

$$
G 1(x)=u(x)+h(x)
$$

for some $h=a x+b$. In view of $u(x)+h(x) \rightarrow-\infty$ as $x \rightarrow 0$ and $x \rightarrow 1$, this contradicts $G 1>0$. Thus $T \in \mathcal{O}_{Q P}^{N}$.

2. Next we show that $\mathcal{O}_{Q P}^{N}<\mathcal{O}_{Q B}^{N} \cap \mathcal{O}_{Q D}^{N}$. Since the boundedness as well as Dirichlet finiteness of $G 1$ trivially implies its existence, we only have to find an $N$-manifold with $Q P \neq \emptyset, Q B=Q D=\emptyset$. We shall henceforth tacitly understand that $\left|y_{i}\right| \leqq 1$ implies identification of opposite faces by pairs.

LEMma 2. The $N$-manifold

$$
T: 0<x<1, \quad\left|y_{i}\right| \leqq 1, \quad i=1, \cdots, N-1,
$$


with the metric

$$
d s^{2}=x^{-3} d x^{2}+x^{3 /(N-1)} \sum_{i=1}^{N-1} d y_{i}^{2}
$$

belongs to $\tilde{\mathcal{O}}_{Q P}^{N} \cap \Theta_{Q B}^{N} \cap \Theta_{Q D}^{N}$ for every $N$.

Proof. Clearly

$$
u=x^{-1} \in Q P,
$$

and the most general $h(x) \in H$ is

Since $G 1$ exists,

$$
h(x)=a x^{-2}+b .
$$

$$
G 1=u+h=a x^{-2}+x^{-1}+c
$$

for some $a, c$. This function is unbounded, hence $T \in \mathcal{O}_{Q B}^{N}$.

Let $D_{x}$ stand for the Dirichlet integral over the subregion of $T$ from $x$ to 1 , and denoted by $c$ a constant, not always the same. Then

$$
\begin{aligned}
& D_{x}(u)=c \int_{x}^{1} x^{-4} x^{3} d x=-c \log x, \\
& D_{x}(h)=c \int_{x}^{1} x^{-6} x^{3} d x=c\left(1-x^{-2}\right),
\end{aligned}
$$

and $D_{x}(G 1)>\left|D_{x}(u)-D_{x}(h)\right|$, which is unbounded as $x \rightarrow 0$. Therefore $T \in \mathcal{O}_{Q D}^{N}$.

3. We proceed to show that $\mathcal{O}_{Q_{B}}^{N} \cap \mathcal{O}_{Q_{D}}^{N}<\mathcal{O}_{Q_{B}}^{N}$.

LEMMA 3. The $N$-manifold

with the metric

$$
T: 0<x<1, \quad\left|y_{i}\right| \leqq 1, \quad i=1, \cdots, N-1,
$$

belongs to $\mathcal{O}_{Q B}^{N} \cap \tilde{\mathcal{O}}_{Q D}^{N}$ for every $N$.

$$
d s^{2}=x^{-2} d x^{2}+x^{2 /(N-1)} \sum_{i=1}^{N-1} d y_{i}^{2}
$$

Proof. We have

with

$$
u=\log x^{-1} \in Q
$$

$$
D(u)=c \int_{0}^{1} x^{-2} x^{2} d x<\infty
$$

Therefore $T \in \widetilde{\mathcal{O}}_{Q D}^{N}$.

The general $h(x) \in H$ is

For some $a, b$,

$$
h=a x^{-1}+b .
$$

$$
G 1=\log x^{-1}+a x^{-1}+b,
$$

which is unbounded. A fortiori $T \in \mathcal{O}_{Q B}^{N}$. 
4. The relation $\mathcal{O}_{Q B}^{N} \cap \mathcal{O}_{Q D}^{N}<\mathcal{O}_{Q D}^{N}$ is known for $N>2$. In fact, by Sario-Wang [8], the Poincaré $N$-ball $B_{\alpha}^{N}: r<1, d s=\left(1-r^{2}\right)^{\alpha}|d x|$, belongs to $\mathcal{O}_{Q B}^{N}$ if and only if $\alpha \notin\left(-1,(N-2)^{-1}\right)$, and to $\mathcal{O}_{Q D}^{N}$ if and only if $\alpha \notin\left(-3(N+2)^{-1},(N-2)^{-1}\right)$. Therefore $B_{\alpha}^{N} \in \widetilde{\mathcal{O}}_{Q B}^{N} \cap \mathcal{O}_{Q D}^{N}$ is characterized by $\alpha \in\left(-1,-3(N+2)^{-1}\right]$. Here we give two other examples, valid for all $N$.

LEMMA 4. The N-manifold

$$
T:|x|<\infty, \quad|y| \leqq 1, \quad\left|z_{\imath}\right| \leqq 1, \quad i=1, \cdots, N-2,
$$

with the metric

$$
d s^{2}=\left(1+x^{4}\right)^{-1} d x^{2}+\left(1+x^{4}\right)\left(1+x^{6}\right)^{2} d y^{2}+\sum_{i=1}^{N-2} d z_{\imath}^{2}
$$

belongs to $\tilde{\mathcal{O}}_{Q B}^{N} \cap \mathcal{O}_{Q D}^{N}$ for every $N$.

Proof. We readily verify that the function

$$
u(x)=-\int_{0}^{x}\left(1+s^{4}\right)^{-1}\left(1+s^{6}\right)^{-1} \int_{0}^{s}\left(1+r^{6}\right) d r d s
$$

belongs to $Q$. As $|x| \rightarrow \infty, u^{\prime}(x) \sim c x^{-3}$ and

Consequently $T \in \widetilde{\mathcal{O}}_{Q B}^{N}$.

$$
u(x)=\mathcal{O}(1) \text {. }
$$

Suppose there exists a $u \in Q D$. Take a function $\varphi_{0} \in C_{0}^{2}((-\infty, \infty)), \varphi_{0} \geqq 0$, supp $\varphi_{0} \subset(0,1)$. For $t>0$, set $\varphi_{t}(x)=\varphi_{0}(x-t)$. Then $\operatorname{supp} \varphi_{t} \subset(t, t+1)$,

and

$$
\left(1, \varphi_{t}\right)=c \int_{t}^{t+1}\left(1+x^{6}\right) \varphi_{t} d x>c t^{6}
$$

$$
D\left(\varphi_{t}\right)=c \int_{t}^{t+1} \varphi_{t}^{\prime 2}\left(1+x^{4}\right)\left(1+x^{6}\right) d x=\mathcal{O}\left(t^{10}\right) .
$$

Therefore $\left(1, \varphi_{t}\right) / \sqrt{D\left(\varphi_{t}\right)} \rightarrow \infty$ as $t \rightarrow \infty$. This violates Stokes' formula, which gives $\left(\Delta u, \varphi_{t}\right)=\left(1, \varphi_{t}\right)<\sqrt{D(u)} \sqrt{D\left(\varphi_{t}\right)}$. We conclude that $T \in \mathcal{O}_{Q D}^{N}$.

5. Our second example for $\widetilde{O}_{Q B}^{N} \cap \Theta_{Q D}^{N}=\emptyset$ is as follows.

LEMma 5. The N-manifold

with the metric

$$
T: 0<x<1, \quad\left|y_{i}\right| \leqq 1, \quad i=1, \cdots, N-1,
$$

$$
d s^{2}=x^{-1} d x^{2}+x^{-5 /(N-1)} \sum_{i=1}^{N-1} d y_{i}^{2}
$$

belongs to $\tilde{\mathcal{O}}_{Q B}^{N} \cap \mathcal{O}_{Q D}^{N}$ for every $N$.

Proof. The general solution of $\Delta u=1$ is

$$
u=a x^{3}+\frac{1}{2} x+b,
$$


which is bounded. Since $G 1=u$ for some $(a, b), T \in \widetilde{\mathcal{O}}_{Q B}^{N}$. By virtue of

$$
D(G 1)=D(u)=c \int_{0}^{1}\left(3 a x^{2}+\frac{1}{2}\right)^{2} x x^{-3} d x=\infty,
$$

we have $T \in \mathcal{O}_{Q D}^{N}$.

6. Trivially $\mathcal{O}_{Q B}^{N} \cup \mathcal{O}_{Q D}^{N} \subset \mathcal{O}_{Q C}^{N}$. If there exist $u \in Q B$ and $v \in Q D$, then $G 1$ is bounded and Dirichlet finite, hence in $Q C$. This gives our last relation $\mathcal{O}_{Q B}^{N} \cup$ $\mathcal{O}_{Q D}^{N}=\mathcal{O}_{Q C}^{N}$.

In the case $N=2$ the relations $\mathcal{O}_{Q P}<\mathcal{O}_{Q B} ; \mathcal{O}_{Q P}<\mathcal{O}_{Q D} ; \mathcal{O}_{Q B} \leftarrow \mathcal{O}_{Q D} ; \mathcal{O}_{Q D} \mp \mathcal{O}_{Q B}$; $\Theta_{Q B}<\Theta_{Q C}$; and $\Theta_{Q D}<\Theta_{Q C}$ can also be established (Nakai-Sario [3]) by means of the identity $\Delta u=\lambda^{-2} \Delta_{e} u$ between the Riemannian $\Delta$, Euclidean $\Delta_{e}$, and the conformal metric $d s=\lambda(z)|d z|$.

7. We collect our results :

THEOREM. The strict inclusion relations

$$
\mathcal{O}_{G}^{N}<\mathcal{O}_{Q P}^{N}<\mathcal{O}_{Q B}^{N} \cap \mathcal{O}_{Q D}^{N}<\mathcal{O}_{Q B}^{N}, \mathcal{O}_{Q D}^{N}<\mathcal{O}_{Q B}^{N} \cup \mathcal{O}_{Q D}^{N}=\mathcal{O}_{Q C}^{N}
$$

between quasiharmonic null classes of Riemannian $N$-manifolds are valid for every dimension $N \geqq 2$.

The author wishes to express his sincere gratitude to Professor Cecilia Wang for a painstaking checking of the manuscript.

\section{BIBLIOGRAPHY}

[1] D. HADA, L. SARIo AND C. WANG, N-manifolds carrying bounded but no Dirichlet finite harmonic functions, Nagoya Math. J. 54 (1974), 1-6.

[2] Y.K. KWON, Strict inclusion $\mathcal{O}_{H B}<\mathcal{O}_{H D}$ for all dimensions, (to appear).

[3] M. NAKAI AND L. SARIO, Quasiharmonic classification of Riemannian manifolds, Proc. Amer. Math. Soc. 31 (1972), 165-169.

[4] M. NAKai and L. Sario, Existence of Dirichlet finite biharmonic functions, Ann. Acad. Sci. Fenn. A. I. 532 (1973), 1-33.

[5] M. NAKai and L. Sario, Existence of bounded biharmonic functions, J. Reine Angew. Math. 259 (1973), 147-156.

[6] L. SARIO, Biharmonic and quasiharmonic functions on Riemannian manifolds, Duplicated lecture notes 1968-70, University of California, Los Angeles.

[7] L. SARIO AND M. NAKaI, Classification Theory of Riemann Surfaces, SpringerVerlag, 1970, 446 pp.

[8] L. SARIO AND C. WANG, Quasiharmonic functions on the Poincaré $N$-ball, Rend. Mat. 6 (1973), 1-14. 